\title{
A reflection on Tingley's problem and some applications
}

\author{
Javier Cabello Sánchez \\ Departamento de Matemáticas, Universidad de Extremadura, Avda. de Elvas s/n, 06006 \\ Badajoz. Spain. Dedicated to the memory of Professor Carlos Benítez.
}

\begin{abstract}
In this paper we show how some metric properties of the unit sphere of a normed space can help to approach a solution to Tingley's problem. In our main result we show that if an onto isometry between the spheres of strictly convex spaces is the identity when restricted to some relative open subset, then it is the identity. This implies that an onto isometry between the unit spheres of strictly convex finite dimensional spaces is linear if and only it is linear on a relative open set. We prove the same for arbitrary two-dimensional spaces and obtain that every two-dimensional, non strictly convex, normed space has the Mazur-Ulam Property.

We also include some other less general, yet interesting, results, along with a generalisation of curvature to normed spaces.
\end{abstract}

Keywords: Mazur-Ulam property, metric invariants, strictly convex spaces, curvature.

2000 MSC: 52A10, 46B04

\section{Introduction}

Since Tingley's seminal paper [1], a lot of work has been done trying to answer this:

Question 1.1. Let $\left(X,\|\cdot\|_{X}\right),\left(Y,\|\cdot\|_{Y}\right)$ be normed spaces and $\tau: S_{X} \rightarrow S_{Y}$ a surjective isometry between their unit spheres. Is $\tau$ the restriction of a linear isometry $\widetilde{\tau}: X \rightarrow Y$ ?

Email address: coco@unex.es (Javier Cabello Sánchez ) 
This Question is widely known as Tingley's problem. The main result in Tingley's paper [1] is what we will call Tingley's Theorem throughout the paper:

Theorem 1.2 (Tingley, [1]). Suppose that $S$ and $S^{\prime}$ are the unit spheres of finite dimensional Banach spaces. If $f: S \rightarrow S^{\prime}$ is an onto isometry, then $f(-x)=-f(x)$ for all $x$ in $S$.

Of course, this is not the first question about the linearity of isometries. Namely, since Mazur-Ulam Theorem, see [2], we are aware of the fact that every surjective isometry $\widetilde{\tau}:\left(X,\|\cdot\|_{X}\right) \rightarrow\left(Y,\|\cdot\|_{Y}\right)$ is affine. The relation between the Theorem and the Question has led to state (see, e.g., [3]) that $\left(X,\|\cdot\|_{X}\right)$ has the Mazur-Ulam Property when the answer to Question 1.1 is affirmative for every $\left(Y,\|\cdot\|_{Y}\right)$. As a consequence of Mazur-Ulam Theorem, Question 1.1 may be replaced by the following:

- Is $\tau$ the restriction of an isometry $\widetilde{\tau}: X \rightarrow Y$ such that $\widetilde{\tau}(0)=0$ ?

The next natural step could have been the question about surjective isometries between unit balls, but Mankiewicz, in [4], showed that in the Mazur-Ulam Theorem the surjective isometry does not need to be defined on the whole space $X$ or even on the unit ball: if we consider two closed convex bodies $F_{X} \subset X$ and $F_{Y} \subset Y$, every onto isometry $\tau: F_{X} \rightarrow F_{Y}$ is affine -it is a little more general, actually. So, Tingley's problem can be then restated as:

- Is $\tau$ the restriction of an isometry $\widetilde{\tau}: B_{X} \rightarrow B_{Y}$ such that $\widetilde{\tau}(0)=0$ ?

It seems that, if every space has the Mazur-Ulam Property, the last question of this kind will be, in the spirit of Mankiewicz's result:

- If $F_{X}$ and $F_{Y}$ are convex bodies and $\tau: \partial F_{X} \rightarrow \partial F_{Y}$ is an onto isometry between their boundaries, does $\tau$ extend linearly?

This problem has been dealt in several ways and lots of positive answers have been found, see, e.g., 15, 6, 7, 8, 9, 10, 11, 12, 13, 14, 15, 16, 17, 18, 19, 20, 21, 22, 23, 24, 25, 26, 27] -it is really impressive the development of machinery and technics that this problem has led to.

Anyway, all usual approaches share a common procedure: take some more o less concrete normed space $\left(X,\|\cdot\|_{X}\right)$ and its unit sphere $S_{X}$, suppose that for some $\left(Y,\|\cdot\|_{Y}\right)$-with or without further assumptions on $\left(Y,\|\cdot\|_{Y}\right)$ - there is some onto isometry $\tau: S_{X} \rightarrow S_{Y}$, analyse some properties of the involved norms and show somehow that the homogeneous extension of $\tau$ is either isometric or linear. This is enough because, in this setting, linear implies 
isometric and vice versa. When we say the homogeneous extension we refer to $\widetilde{\tau}: X \rightarrow Y$ defined as $\widetilde{\tau}(\lambda x)=\lambda \tau(x)$ for every $\lambda \geq 0, x \in S_{X}$.

Our approach will follow a different way: if $\tau$ is the restriction of a linear isometry, in particular $\tau$ must be linear. We mean that, whenever $x, x^{\prime} \in S_{X}$ and $\lambda, \lambda^{\prime} \in \mathbb{R}$ are such that $\lambda x+\lambda^{\prime} x^{\prime} \in S_{X}$, the point $\lambda \tau(x)+\lambda^{\prime} \tau\left(x^{\prime}\right)$ must belong to $S_{Y}$ and the equality

$$
\tau\left(\lambda x+\lambda^{\prime} x^{\prime}\right)=\lambda \tau(x)+\lambda^{\prime} \tau\left(x^{\prime}\right)
$$

must hold. So, taking coordinates with respect to well chosen bases, we will have that if $\tau$ is the restriction of some linear isometry then its representation in coordinates must be the identity. Of course, the identity of a sphere is the restriction of a linear isometry, so this is a necessary and sufficient condition for $\tau$ to be the restriction of a linear isometry.

As our immediate goal is not to detail thoroughly our method, we will try to explain it in its simplest form. Consider some two-dimensional normed space $\left(X,\|\cdot\|_{X}\right)$ and suppose that there is a surjective isometry $\tau: S_{X} \rightarrow S_{Y}$ for some $\left(Y,\|\cdot\|_{Y}\right)$-it must be two-dimensional, too. We may take a basis $\mathcal{B}_{X}=\left\{x_{1}, x_{2}\right\} \subset S_{X}$ and identify linearly isometrically $\left(X,\|\cdot\|_{X}\right)$ with $\left(\mathbb{R}^{2},\|\cdot\|_{X}^{\prime}\right)$ and $\mathcal{B}_{X}$ with the usual basis $\mathcal{B}_{2}=\left\{e_{1}, e_{2}\right\}$ in $\mathbb{R}^{2}$ :

$$
\phi_{X}\left(\lambda_{1} x_{1}+\lambda_{2} x_{2}\right)=\left(\lambda_{1}, \lambda_{2}\right), \quad\left\|\left(\alpha_{1}, \alpha_{2}\right)\right\|_{X}^{\prime}=\left\|\alpha_{1} x_{1}+\alpha_{2} x_{2}\right\|_{X} .
$$

If we take, further, $y_{1}=\tau\left(x_{1}\right), y_{2}=\tau\left(x_{2}\right)$ and $\mathcal{B}_{Y}=\left\{y_{1}, y_{2}\right\} \subset S_{Y}$, then $\mathcal{B}_{Y}$ is a basis of $Y$ by Tingley's Theorem. Identifying the same way $Y$ with $\mathbb{R}^{2}$ and $\mathcal{B}_{Y}$ with $\mathcal{B}_{2}$ via $\phi_{Y}: Y \rightarrow \mathbb{R}^{2}$ we may consider the map $\tau^{\prime}: S_{\|\cdot\|_{X}^{\prime}} \subset \mathbb{R}^{2} \rightarrow$ $S_{\|\cdot\|_{Y}^{\prime}} \subset \mathbb{R}^{2}$ defined as $\tau^{\prime}\left(\lambda_{1}, \lambda_{2}\right)=\left(\mu_{1}, \mu_{2}\right)$ when $\tau\left(\lambda_{1} x_{1}+\lambda_{2} x_{2}\right)=\mu_{1} y_{1}+\mu_{2} y_{2}$, i.e., $\tau^{\prime}=\phi_{Y} \circ \tau \circ \phi_{X}^{-1}$. As $\tau^{\prime}(1,0)=(1,0)$ and $\tau^{\prime}(0,1)=(0,1)$, the only way $\tau^{\prime}$ can be linearly extended is being the identity so, since $\phi_{X}$ and $\phi_{Y}$ are linear, we have two options:

- If $\tau^{\prime}$ is the identity then $\tau: S_{X} \rightarrow S_{Y}$ is the restriction of a linear isometry.

- If $\tau^{\prime}$ is not the identity then there is no linear application whose restriction agrees with $\tau$.

So, for $\widetilde{\tau}: X \rightarrow Y$ to be linear it is necessary $S_{\|\cdot\|_{X}^{\prime}}=S_{\|\cdot\|_{Y}^{\prime}}$. We will see that this is also sufficient in two-dimensional spaces, so the planar Tingley's problem could be stated as follows: 
Question 1.3. Suppose $\|\cdot\|_{X}$ and $\|\cdot\|_{Y}$ are two norms defined on $\mathbb{R}^{2}$ such that $\|(1,0)\|=\|(0,1)\|=1$ for both norms and there is an isometry $\tau: S_{X} \rightarrow S_{Y}$ such that $\tau(1,0)=(1,0), \tau(0,1)=(0,1)$. Does this imply $S_{X}=S_{Y}$ ?

\subsection{Notations}

Throughout this paper, $X$ and $Y$ will be normed spaces. When we deal with more than one space, we will write $\|\cdot\|_{X},\|\cdot\|_{Y}$ and so on for the norms unless we are referring, on purpose, to equalities or relations that hold for all the involved norms, as in Question 1.3.

We will denote by $\mathcal{B}_{n}=\left\{e_{1}, \ldots, e_{n}\right\}$ the usual basis of $\mathbb{R}^{n}$, in particular every appearance of $e_{i}$ will refer to the $i$-th vector of $\mathcal{B}_{n}$.

We have avoided the use of open intervals or segments, so that the notation $(a, b)$ will always refer to a two-dimensional vector. For closed intervals or segments, we will write $\left[x, x^{\prime}\right]$, i.e, $\left[x, x^{\prime}\right]=\left\{\lambda x+\left(1-\lambda x^{\prime}\right): 0 \leq \lambda \leq 1\right\}$ no matter whether $x$ and $x^{\prime}$ are scalars or vectors.

Given $x, x^{\prime} \in X$ we will denote as $\operatorname{Bis}\left(x, x^{\prime}\right)$ the bisector of the segment $\left[x, x^{\prime}\right]$, i.e., $\operatorname{Bis}\left(x, x^{\prime}\right)=\left\{z \in X:\|x-z\|_{X}=\left\|x^{\prime}-z\right\|_{X}\right\}$. As we will deal frequently with bisectors of symmetric segments of the form $\operatorname{Bis}(x,-x)$, we will refer to them as symmetric bisectors and will omit the $-x$ in the notation, so $\operatorname{Bis}(x)$ will be the symmetric bisector of $x$ and must be understood as $\operatorname{Bis}(-x, x)$. Please observe that $z \in \operatorname{Bis}(x)$ if and only if $x$ and $z$ are isosceles orthogonal.

Definition 1.4. Given a segment $\left[x, x^{\prime}\right]$ in the sphere of some two-dimensional normed space $\left(X,\|\cdot\|_{X}\right)$, we say that $\left[x, x^{\prime}\right]$ is maximal when it is not strictly contained in another segment.

Definition 1.5. For $x \in S_{X}$, the star of $x$ is $\left\{x^{\prime} \in S_{X}:\left[x, x^{\prime}\right] \subset S_{X}\right\}$.

As we will focus primarily on metric concepts, the following subset will play the usual role of the star. The definition of the star is here just for, say, compatibility purposes.

Definition 1.6. Given $x \in S_{X}$, we will denote by $\mathcal{D}(x)$ the set $\left\{x^{\prime} \in S_{X}\right.$ : $\left.\left\|x-x^{\prime}\right\|_{X}=2\right\}$.

Remark 1.7. By Corollary 5 in [1], $\mathcal{D}(x)$ agrees with the star of $-x$, so $\mathcal{D}(x)=\left\{x^{\prime} \in S_{X}:\left[-x,-x^{\prime}\right] \subset S_{X}\right\}$. 


\subsection{Plan of the paper}

Apart from this Introduction, the paper is divided into three sections.

The first one is devoted to some quite elementary, general facts about spheres in normed spaces. These facts will be useful in the remaining two sections.

Section 3 is the central one, we have split the proof of the main results into several parts. Some of these intermediate results are interesting on their own, and also some proofs reveal the main ideas in this paper far better than the main results' proofs.

Finally, in Section 4 we expose some results than can be seen as consequences of the ideas more than consequences of the results in Section 3. It includes a subsection where we define a kind of generalisation of the usual curvature of planar curves.

\section{The general results}

Given a finite dimensional $\left(X,\|\cdot\|_{X}\right)$ and a basis $\mathcal{B}_{X}=\left\{x_{1}, \ldots, x_{n}\right\} \subset X$, we say that $\left(\left(X,\|\cdot\|_{X}\right), \mathcal{B}_{X}\right)$ is identified with $\left(\mathbb{R}^{n},\|\cdot\|_{X}^{\prime}\right)$ if

$$
\left\|\left(\lambda_{1}, \ldots, \lambda_{n}\right)\right\|_{X}^{\prime}=\left\|\lambda_{1} x_{1}+\cdots \lambda_{n} x_{n}\right\|_{X}
$$

for every $\left(\lambda_{1}, \ldots, \lambda_{n}\right) \in \mathbb{R}^{n}$, i.e., if the linear application $\phi_{X}:\left(X,\|\cdot\|_{X}\right) \rightarrow$ $\left(\mathbb{R}^{n},\|\cdot\|_{X}^{\prime}\right)$ given by $\phi_{X}\left(x_{i}\right)=e_{i}, i=1, \ldots, n$ is an isometry.

Lemma 2.1. Let $\left(X,\|\cdot\|_{X}\right),\left(Y,\|\cdot\|_{Y}\right)$ be finite dimensional normed spaces, $\tau: S_{X} \rightarrow S_{Y}$ an application (respectively, isometry), and suppose that $\mathcal{B}_{X}=$ $\left\{x_{1}, \ldots, x_{n}\right\} \subset S_{X}$ and $\mathcal{B}_{Y}=\left\{\tau\left(x_{1}\right), \ldots, \tau\left(x_{n}\right)\right\} \subset S_{Y}$ are bases of $X$ and $Y$ respectively. Suppose, moreover, that $\left(\left(X,\|\cdot\|_{X}\right), \mathcal{B}_{X}\right)$ and $\left(\left(Y,\|\cdot\|_{Y}\right), \mathcal{B}_{Y}\right)$ are identified with $\left(\mathbb{R}^{n},\|\cdot\|_{X}^{\prime}\right)$ and $\left(\mathbb{R}^{n},\|\cdot\|_{Y}^{\prime}\right)$ via $\phi_{X}$ and $\phi_{Y}$. Then, $\tau$ is the restriction of a linear application (resp, isometry) $\widetilde{\tau}: X \rightarrow Y$ if and only if $\tau^{\prime}=\phi_{Y} \circ \tau \circ \phi_{X}^{-1}: S_{\|\cdot\|_{X}^{\prime}} \rightarrow S_{\|\cdot\|_{Y}^{\prime}}$ is the identity.

Proof. Since $\phi_{X}$ and $\phi_{Y}$ are onto linear isometries and $\tau^{\prime}\left(e_{i}\right)=e_{i}$ for every $i$, the results follows.

Definition 2.2. We say that a norm $\|\cdot\|_{X}$ defined on $\mathbb{R}^{n}$ is normalized if $\left\|e_{i}\right\|_{X}=1$ for every $e_{i} \in \mathcal{B}_{n}$.

Theorem 2.3. Let $\|\cdot\|_{X},\|\cdot\|_{Y}$ be normalized norms defined on $\mathbb{R}^{2}, \tau: S_{X} \rightarrow$ $S_{Y}$ an isometry such that $\tau(1,0)=(1,0)$ and $\tau(0,1)=(0,1)$. Then $\tau$ is the restriction of a linear isometry if and only if $S_{X}=S_{Y}$. 
Proof. Let $\tau$ be as in the statement. By Lemma 2.1, if $S_{X}$ and $S_{Y}$ are different, then $\tau$ is not linear, so the "only if" part is done. What we need to show in order to prove the other implication is that $S_{Y}=S_{X}$ implies that the only isometry $\tau: S_{X} \rightarrow S_{Y}$ is the identity.

Take some $z, \tau(z) \in S_{X}$. As $\tau$ preserves distances and $\tau\left(e_{1}\right)=e_{1}, \tau\left(e_{2}\right)=$ $e_{2}$, we have $\left\|z \pm e_{1}\right\|=\left\|\tau(z) \pm e_{1}\right\|$, and $\left\|z \pm e_{2}\right\|=\left\|\tau(z) \pm e_{2}\right\|$. The Monotonicity lemma (see, e.g., [28], Proposition 31) implies that for any normed plane $E$ and any basis $\left\{u_{1}, u_{2}\right\} \subset E$, the four distances $\left\|v \pm u_{1}\right\|_{E}$, $\left\|v \pm u_{2}\right\|_{E}$ determine $v$ when $v, u_{1}, u_{2} \in S_{E}$, so we have $z=\tau(z)$.

Remark 2.4. This result is very similar to [29], Corollary 2.12.

Conjecture 2.5. Theorem 2.3 is true for any couple of normalized norms defined on $\mathbb{R}^{n}$.

Of course this conjecture is a particular case of Tingley's Problem, we explicited it here just because it seems much easier to answer and it could be helpful. See Remark 3.10 for a little further explanation.

Remark 2.6. The proof of Theorem 2.3 will not adapt to this Conjecture, here we explicit a simple example of a three-dimensional space with a basis that does not determine the points in the sense used above. Take $\left(\mathbb{R}^{3},\|\cdot\|_{\infty}\right)$ and the basis $\left\{v_{1}, v_{2}, v_{3}\right\}=\{(1,1,1),(1,1,0.9),(1,0.9,1)\}$. We need to point out that all the coordinates in this Remark refer to the usual basis. It is clear that, for $y_{1}=(1,-1,0.1), y_{2}=(1,-1,-0.1)$, we have

- $\left\|v_{i}+y_{j}\right\|=2$ for every $i, j$.

- $\left\|v_{i}-y_{j}\right\|=2$ if $i, j \in\{1,2\}$.

- $\left\|v_{3}-y_{j}\right\|=1.9$ for $j \in\{1,2\}$.

On the other hand, it is not hard to see that the only isometry of $S_{\infty}^{3}$ that preserves $\left\{v_{1}, v_{2}, v_{3}\right\}$ is the identity. Indeed, suppose $\tau: S_{\infty}^{3} \rightarrow S_{\infty}^{3}$ is an onto isometry and $\tau\left(v_{i}\right)=v_{i}, i=1,2,3$.

Then, $\tau$ preserves $\mathcal{D}\left(v_{1}\right) \cap \mathcal{D}\left(v_{2}\right) \cap \mathcal{D}\left(v_{3}\right)=\{-1\} \times[-1,1] \times[-1,1]$, so it preserves $\{1\} \times[-1,1] \times[-1,1]$, too. Now it is clear that $\tau$ also preserves

$$
\left(\mathcal{D}\left(v_{1}\right) \cap \mathcal{D}\left(v_{2}\right)\right) \backslash\left(\mathcal{D}\left(v_{1}\right) \cap \mathcal{D}\left(v_{2}\right) \cap \mathcal{D}\left(v_{3}\right)\right)=\operatorname{int}([-1,1] \times\{-1\} \times[-1,1])
$$

and also $[-1,1] \times[-1,1] \times\{-1\}$, so $\tau(C)=C$ when $C$ is any of the six faces of the unit sphere. It is clear that every $\left(a_{1}, a_{2}, a_{3}\right)$ belonging to the ball 
$B_{\infty}^{3}$ is determined by its distances to the six faces of $S_{\infty}^{3}$, so $\tau\left(a_{1}, a_{2}, a_{3}\right)=$ $\left(a_{1}, a_{2}, a_{3}\right)$, for every $\left(a_{1}, a_{2}, a_{3}\right) \in S_{\infty}^{3}$.

We can explicit another example, this one involves a strictly convex space. Consider $\left(\mathbb{R}^{3},\|\cdot\|_{3}\right)$ and take $x=\frac{1}{\sqrt[3]{3}}(1,1,1) \in S_{3}^{3}$. Then,

$$
v_{1}=\frac{1}{\sqrt[3]{6}}(1,1,-\sqrt[3]{4}), v_{2}=\frac{1}{\sqrt[3]{6}}(1,-\sqrt[3]{4}, 1), v_{3}=\frac{1}{\sqrt[3]{6}}(-\sqrt[3]{4}, 1,1)
$$

form a basis such that $\left\|v_{i}-x\right\|_{3}=\left\|v_{j}+x\right\|_{3}$ for $i, j \in\{1,2,3\}$, so this basis does not distinguish $x$ and $-x$. Indeed it is easy to check that all these quantities equal $\left(\frac{4}{3}+2 \sqrt[3]{2}\right)^{1 / 3}$. What we have done is to choose the simplest $x \in S_{3}^{3}$ whose symmetric bisector is not planar and the simplest basis contained in its symmetric bisector: $v_{1}, v_{2}, v_{3} \in \operatorname{Bis}(x)$. Now, the following seems pretty natural:

Question 2.7. Let $\left(X,\|\cdot\|_{X}\right)$ be a three-dimensional normed space. Can we choose $x, v_{1}, v_{2}, v_{3} \in S_{X}$ such that $\left\{v_{1}, v_{2}, v_{3}\right\}$ is a basis and $v_{1}, v_{2}, v_{3} \in \operatorname{Bis}(x)$ whenever $X$ is not Euclidean?

Remark 2.8. It is clear from [30], Theorem 3.2, that in every not Euclidean three-dimensional space $\left(X,\|\cdot\|_{X}\right)$, for each $\lambda \in(0,1) \cup(1, \infty)$ there exist $x, v_{1}, v_{2}, v_{3} \in S_{X}$ such that $v_{1}, v_{2}, \lambda v_{3} \in \operatorname{Bis}(x)$ and $\left\{v_{1}, v_{2}, v_{3}\right\}$ is a basis.

\section{The main results}

We have tried to explicit every useful property, so we have split the proof of the main results into several intermediate steps.

Remark 3.1. Consider an onto isometry $\tau: S_{X} \rightarrow S_{Y}$ between the spheres of a pair of finite dimensional normed spaces. By Tingley's Theorem, for every $x \in S_{X}$ we have $\tau(-x)=-\tau(x)$, so

$\left\|\tau\left(x_{1}\right)+\tau\left(x_{2}\right)\right\|_{Y}=\left\|\tau\left(x_{1}\right)-\left(-\tau\left(x_{2}\right)\right)\right\|_{Y}=\left\|\tau\left(x_{1}\right)-\tau\left(-x_{2}\right)\right\|_{Y}=\left\|x_{1}-\left(-x_{2}\right)\right\|_{X}=\left\|x_{1}+x_{2}\right\|_{X}$,

and this readily implies that $\tau\left(x_{1}\right)$ and $\tau\left(x_{2}\right)$ belong to the same segment if and only if $x_{1}$ and $x_{2}$ do.

Definition 3.2. For $x \in S_{X}$, we say that $x$ is flat if there is an affine hyperplane $H \subset X$ such that $H \cap S_{X}$ is a relative neighbourhood of $x$.

Observation 3.3. Let $x \in S_{X}$. Then, $x$ is flat if and only if $\mathcal{D}(x)=\mathcal{D}\left(x^{\prime}\right)$ for every $x^{\prime} \in S_{X}$ in a relative neighbourhood of $x$. As a consequence, being flat is an intrinsic metric property for points in $S_{X}$. 
Proof. By Remark 1.7, if $H \cap S_{X}$ contains a (nonempty) relative open subset, say $U \cap S_{X}=\operatorname{int}_{S_{X}}\left(H \cap S_{X}\right)$, then $\mathcal{D}(x)=-H \cap S_{X}$, for every $x \in U$. In particular, $\mathcal{D}(x)=\mathcal{D}\left(x^{\prime}\right)$ for $x^{\prime} \in U$.

On the other hand, let $x \in S_{X}$ and suppose $\mathcal{D}(x)=\mathcal{D}\left(x^{\prime}\right)$ for every $x^{\prime} \in U$, where $U=S_{X} \cap\left(x+\varepsilon B_{X}\right)$. By Remark 1.7, $\mathcal{D}(x)=\mathcal{D}\left(x^{\prime}\right)$ if and only if

$$
\left\{\bar{x} \in S_{X}:[x, \bar{x}] \subset S_{X}\right\}=\left\{\bar{x} \in S_{X}:\left[x^{\prime}, \bar{x}\right] \subset S_{X}\right\} .
$$

Then, $\left[x^{\prime}, \bar{x}\right] \subset S_{X}$ for every $x^{\prime}, \bar{x} \in U$. This implies that $U$ is convex, so it is contained in $S_{X} \cap H$ for some hyperplane $H$. This means that $S_{X} \cap H$ is also a relative neighbourhood of $x$.

The following result has been recently published as Theorem 2.6 in [27]. We include it here because we think that our proof is interesting enough.

Proposition 3.4 (Wang, Huang, [27, Theorem 2.6]). Let $\left(X,\|\cdot\|_{X}\right)$ be a two-dimensional normed space whose unit sphere contains a segment with length at least 1. Then, $\left(X,\|\cdot\|_{X}\right)$ has the Mazur-Ulam Property.

Proof. Suppose that $\left(X,\|\cdot\|_{X}\right)$ is such a space and suppose there are some $\left(Y,\|\cdot\|_{Y}\right)$ and $\tau: S_{X} \rightarrow S_{Y}$ such that $\tau$ is an onto isometry. Let $\left[x_{1}, x_{2}\right]$ be a maximal segment in $S_{X}$ such that $\left\|x_{1}-x_{2}\right\|_{X} \geq 1$. Since $x_{1}, x_{2}$ are not flat points, Observation 3.3 implies that neither $y_{1}=\tau\left(x_{1}\right), y_{2}=\tau\left(x_{2}\right)$ are, but Remark 3.1 implies that $\left[y_{1}, y_{2}\right] \subset S_{Y}$ and we deduce that $\left[y_{1}, y_{2}\right]$ is a maximal segment in $S_{Y}$. As $\left\|y_{1}-y_{2}\right\|_{Y}=\left\|x_{1}-x_{2}\right\|_{X} \geq 1$, the sphere $S_{Y}$ contains another segment with length at least 1 .

Take $\mathcal{B}_{X}=\left\{u_{1}, u_{2}\right\}$ as a basis of $X$, where $u_{1}$ and $u_{2}$ are $u_{1}=\frac{1}{\left\|x_{1}-x_{2}\right\|_{X}}\left(x_{1}-\right.$ $\left.x_{2}\right)$ and $u_{2}=\frac{1}{2}\left(x_{1}+x_{2}\right)$. Consider $Y$ endowed with the analogous basis, $\mathcal{B}_{Y}=\left\{v_{1}, v_{2}\right\}$ given by $v_{1}=\frac{1}{\left\|y_{1}-y_{2}\right\|_{Y}}\left(y_{1}-y_{2}\right)$ and $v_{2}=\frac{1}{2}\left(y_{1}+y_{2}\right)$. We will make heavy use of coordinates, so please recall that they will refer to these bases for the remainder of the proof.

Denoting $\lambda=\frac{1}{2}\left\|x_{1}-x_{2}\right\|_{X}=\frac{1}{2}\left\|y_{1}-y_{2}\right\|_{Y} \geq \frac{1}{2}$, we have $x_{1}=(\lambda, 1), x_{2}=$ $(-\lambda, 1) \in S_{X}$ and $y_{1}=(\lambda, 1), y_{2}=(-\lambda, 1) \in S_{Y}$. Both spheres include the segment $[-\lambda, \lambda] \times\{1\}$ and so, its opposite $[-\lambda, \lambda] \times\{-1\}$. In both spaces we have $\|(1,0)\|=1$, so we actually have $([-\lambda, \lambda] \times\{-1,1\}) \cup\{( \pm 1,0)\} \subset S$. As $\tau(\lambda, 1)=(\lambda, 1)$ and $\tau(-\lambda, 1)=(-\lambda, 1)$, Mankiewicz Theorem implies that $\tau(\alpha, 1)=(\alpha, 1)$ when $\alpha \in[-\lambda, \lambda]$ and, by Tingley's Theorem, we also have $\tau(-\alpha,-1)=(-\alpha,-1)$ for every $\alpha \in[-\lambda, \lambda]$.

Summing up all these data, the convexity of the unit ball of any norm implies that every remaining point of each sphere lies inside some of the 
following four triangles. If $(\alpha, \beta)$ belongs to any of the spheres -and not to their above described subsets- then:

- $\alpha, \beta \geq 0$ implies $(\alpha, \beta) \in \operatorname{conv}\{(\lambda, 1),(2-\lambda, 1),(1,0)\}$.

- $\alpha \geq 0, \beta \leq 0$ implies $(\alpha, \beta) \in \operatorname{conv}\{(\lambda,-1),(2-\lambda,-1),(1,0)\}$.

- $\alpha \leq 0, \beta \geq 0$ implies $(\alpha, \beta) \in \operatorname{conv}\{(-\lambda, 1),(\lambda-2,1),(-1,0)\}$.

- $\alpha, \beta \leq 0$ implies $(\alpha, \beta) \in \operatorname{conv}\{(-\lambda,-1),(\lambda-2,-1),(-1,0)\}$.

We may suppose $\alpha, \beta \geq 0$, being the other cases symmetric. As $\lambda \in$ $[1 / 2,1]$, we have $(\alpha, \beta) \in \operatorname{conv}\{(1 / 2,1),(3 / 2,1),(1,0)\}$. We shall see that there are two more metric-depending parameters that determine both $\alpha$ and $\beta$-and determined by $\alpha$ and $\beta$. Indeed, as the upmost part of both spheres consist of the segment $[(-\lambda, 1),(\lambda, 1)]$, for each point $(a, b) \in \mathbb{R}^{2}$ there is a cone where the distances to $(a, b)$ are just the differences between their second coordinates. Namely, if $\left(a^{\prime}, b^{\prime}\right)$ is such that $\left|\left(a-a^{\prime}\right)\right| \leq \lambda\left|\left(b-b^{\prime}\right)\right|$, then $\left\|(a, b)-\left(a^{\prime}, b^{\prime}\right)\right\|_{X}=\left\|(a, b)-\left(a^{\prime}, b^{\prime}\right)\right\|_{Y}=\left|b-b^{\prime}\right|$. So, the distance from a given $(\alpha, \beta) \in \operatorname{conv}\{(1 / 2,1),(3 / 2,1),(1,0)\}$ to $(1 / 2,-1)$ is precisely $1+\beta$.

This implies that the point $(\alpha, \beta) \in S$ at distance $1 \leq d<2$ from $(1 / 2,-1)$ and distance smaller than 1 from $(1 / 2,1)$ is $(\alpha, d-1)$ for some $\alpha \geq \lambda$, so the second coordinate of $\tau(\alpha, \beta)$ is $\beta$.

We may determine $\alpha$ by means of the metric, too. Indeed, fix $\beta \in[0,1]$ and take $1-\beta / 2 \leq \alpha \leq 1+\beta / 2$, so that $(\alpha, \beta) \in \operatorname{conv}\{(1 / 2,1),(3 / 2,1),(1,0)\}$. Let $(\delta,-1)$ be the leftmost point in the intersection of $\mathbb{R} \times\{-1\}$ and $(\alpha, \beta)+$ $(1+\beta) S$, it is straightforward that it is also the leftmost point in the intersection of $\mathbb{R} \times\{-1\}$ and the cone $\left\{(a, b) \in \mathbb{R}^{2}:|a-\alpha| \leq \lambda|b-\beta|\right\}$. It is clear that the inequality that defines the cone is an equality for $(\delta,-1)$, so $\delta$ fulfils $|(\delta-\alpha) /(-1-\beta)|=\lambda$ and it is obvious that $\delta<\alpha$, so

$$
(\delta-\alpha) /(-1-\beta)=\lambda \text {. }
$$

As $(\delta,-1)$ is fixed, this means that $\|\cdot\|_{X}$ determines $\alpha$. Namely, $\alpha=\delta+$ $(1+\beta) \lambda$ where $\beta, \delta$ and $\lambda$ just depend on distances that agree for $\|\cdot\|_{X}$ and $\|\cdot\|_{Y}$. This means that the only possibility is that $\tau(\alpha, \beta)$ is again $(\alpha, \beta)$.

Corollary 3.5. $\mathbb{R}^{2}$, endowed with any polygonal norm, has the Mazur-Ulam Property. 
Proof. This is just the simplest case of the main result in [16], but here we explicit a proof based on the proof of Proposition 3.4.

Let $\left(X,\|\cdot\|_{X}\right)$ be such that $S_{X}$ is a polygon and take some segment $H_{0}=\left[x_{0}, x_{1}\right] \subset S_{X}$ and $\lambda=\frac{1}{2}\left\|x_{1}-x_{0}\right\|_{X}$. Take another segment $H_{1}=$ $\left[x_{1}, x_{2}\right] \subset S_{X}$, adjacent to $H_{0}$, and suppose that there exist $\left(Y,\|\cdot\|_{Y}\right)$ and $\tau: S_{X} \rightarrow S_{Y}$ such that $\tau$ is an onto isometry. Consider on $X$ the basis

$$
\mathcal{B}_{X}=\left\{\left(x_{1}-x_{0}\right) /\left\|x_{1}-x_{0}\right\|_{X},\left(x_{1}+x_{0}\right) / 2\right\} .
$$

As before, we have $\|(1,0)\|_{X}=1, x_{1}=(\lambda, 1)$ and $x_{0}=(-\lambda, 1)$. If we take the basis

$$
\mathcal{B}_{Y}=\left\{\left(\tau\left(x_{1}\right)-\tau\left(x_{0}\right)\right) /\left\|\tau\left(x_{1}\right)-\tau\left(x_{0}\right)\right\|_{Y},\left(\tau\left(x_{1}\right)+\tau\left(x_{0}\right)\right) / 2\right\},
$$

then $\|(1,0)\|_{Y}=1$ and we may apply verbatim the argument in the previous proof to obtain

$$
\tau(\alpha, 1)=(\alpha, 1), \forall \alpha \in[-\lambda, \lambda]
$$

and also $\tau(\alpha, \beta)=(\alpha, \beta)$ whenever $\alpha \in[\lambda, 2 \lambda]$ and $\beta>0$. This means that there is some $(\alpha, \beta) \in H_{1},(\alpha, \beta) \neq(\lambda, 1)$ such that $\tau(\alpha, \beta)=(\alpha, \beta)$. By Mankiewicz Theorem, this implies that every point in $H_{1}$ is fixed.

Of course, if we now rotate both $S_{X}$ and $S_{Y}$ by taking as bases

$$
\begin{gathered}
\mathcal{B}_{X}^{\prime}=\left\{\left(x_{2}-x_{1}\right) /\left\|x_{2}-x_{1}\right\|_{X},\left(x_{2}+x_{1}\right) / 2\right\} \text { and } \\
\mathcal{B}_{Y}^{\prime}=\left\{\left(\tau\left(x_{2}\right)-\tau\left(x_{1}\right)\right) /\left\|\tau\left(x_{2}\right)-\tau\left(x_{1}\right)\right\|_{Y},\left(\tau\left(x_{2}\right)+\tau\left(x_{1}\right)\right) / 2\right\}
\end{gathered}
$$

then both rotations have the same expression in coordinates, so $\tau$ is still the identity on $H_{0} \cup H_{1}$. Applying the same reasoning to $H_{2}=\left[x_{2}, x_{3}\right] \subset S_{X}$ and so on, we obtain that $\tau$ is the identity on $S_{X}$.

Definition 3.6. When in two-dimensional spaces, and given a couple of linearly independent $x, x^{\prime} \in S_{X}$, the arc that connects $x$ and $x^{\prime}$ is defined as

$$
A\left(x, x^{\prime}\right)=\left\{\lambda x+\lambda^{\prime} x^{\prime}: \lambda, \lambda^{\prime} \geq 0\right\} \cap S_{X}
$$

and it is the smallest connected subset of $S_{X}$ that contains both $x$ and $x^{\prime}$.

Theorem 3.7. Let $\left(X,\|\cdot\|_{X}\right),\left(Y,\|\cdot\|_{Y}\right)$ be two-dimensional normed spaces for which there exists an onto isometry $\tau: S_{X} \rightarrow S_{Y}$. If there is some relative open $U \subset S_{X}$ where $\tau$ is linear, then $\tau$ is linear on $S_{X}$. 
Proof. Suppose there is an arc $H=A\left(x_{1}, x_{2}\right) \subset S_{X}$ such that

$$
\tau\left(\lambda_{1} x_{1}+\lambda_{2} x_{2}\right)=\lambda_{1} \tau\left(x_{1}\right)+\lambda_{2} \tau\left(x_{2}\right)
$$

for every positive $\lambda_{1}, \lambda_{2}$ for which $\lambda_{1} x_{1}+\lambda_{2} x_{2} \in S_{X}$. We will show that $H$ is contained in another arc that fulfils the same condition. As $S_{X}$ is compact, this is enough.

If $H$ contains some segment $\left[x^{\prime}, x_{1}\right]$, then the argument in the previous proofs shows that $\tau$ is linear in a relative neighbourhood of $x_{1}$, so we may suppose that $\mathcal{D}\left(x_{1}\right) \cap(-H)=\left\{-x_{1}\right\}$. Taking, as usual, $\lambda=\frac{1}{2}\left\|x_{2}-x_{1}\right\|_{X}$,

$$
\begin{gathered}
\mathcal{B}_{X}=\left\{\left(x_{2}-x_{1}\right) /\left\|x_{2}-x_{1}\right\|_{X},\left(x_{2}+x_{1}\right) / 2\right\} \text { and } \\
\mathcal{B}_{Y}=\left\{\left(\tau\left(x_{2}\right)-\tau\left(x_{1}\right)\right) /\left\|\tau\left(x_{2}\right)-\tau\left(x_{1}\right)\right\|_{Y},\left(\tau\left(x_{2}\right)+\tau\left(x_{1}\right)\right) / 2\right\},
\end{gathered}
$$

we may pass to coordinates to obtain that $\tau$ is the identity on the arc lying between $(-\lambda, 1)$ and $(\lambda, 1)$. Of course, $\tau$ is also the identity on the opposite arc and this implies, in particular, that $\tau(\lambda,-1)=(\lambda,-1)$.

Let $\mu=\frac{1}{\|(0,1)\|}$, so that $(0, \mu) \in S_{X} \cap S_{Y}$ and $\mu>1$, and observe that the points in $H$, along with the distances between them, determine the shape of $S_{X}$ (and $\left.S_{Y}\right)$ in a relative neighbourhood of $(1,0)$. In particular, for $z \in[0,2 \lambda] \times[3-2 \mu, 2 \mu-1]$, we have

$$
\|z-(-\lambda, 1)\|_{X}=\|z-(-\lambda, 1)\|_{Y}
$$

because $z-(-\lambda, 1)$ is close to the horizontal axis. In the same way, as $S_{X}$ and $S_{Y}$ coincide near $(0, \mu)$, we have

$$
\|z-(\lambda,-1)\|_{X}=\|z-(\lambda,-1)\|_{Y}
$$

because $z-(\lambda,-1)$ is close to the vertical axis.

So, if $z=\left(z_{1}, z_{2}\right) \in([0,2 \lambda] \times[3-2 \mu, 2 \mu-1]) \cap\left(S_{X} \backslash H\right)$, then

$$
\begin{gathered}
a=\|\tau(z)-(\lambda,-1)\|_{Y}=\|z-(\lambda,-1)\|_{X}=\|z-(\lambda,-1)\|_{Y} \text { and } \\
b=\|\tau(z)-(-\lambda, 1)\|_{Y}=\|z-(-\lambda, 1)\|_{X}=\|z-(-\lambda, 1)\|_{Y} .
\end{gathered}
$$

As $(\lambda,-1)$ does not lie in the interior of a segment included is $S_{Y}$, there are only two points in $\left((\lambda,-1)+a S_{Y}\right) \cap\left((-\lambda, 1)+b S_{Y}\right)$. Namely, one of these points is $z$ and the other one, say $z^{\prime}$, lies at the other side of the line 
$\{t \cdot(\lambda,-1): t \in \mathbb{R}\}$. So, the only possibilities are $\tau(z)=z$ or $\tau(z)=z^{\prime}$. But the Monotonicity Lemma implies that

$$
\left\|z^{\prime}-(\lambda, 1)\right\| \geq \min \{\|(\lambda,-1)-(\lambda, 1)\|,\|(\lambda,-1)-(\lambda, 1)\|\} .
$$

So, assuming that

$$
\|z-(\lambda, 1)\|_{X} \leq \min \{\|(\lambda,-1)-(\lambda, 1)\|,\|(\lambda,-1)-(\lambda, 1)\|\},
$$

which we clearly can do, the previous reasonings lead to $\tau(z)=z$ and we have finished the proof.

Corollary 3.8. Every two-dimensional, non strictly convex, normed space has the Mazur-Ulam Property.

Proof. Suppose $\left(X,\|\cdot\|_{X}\right)$ is a two-dimensional normed space and $\left[x, x^{\prime}\right] \subset$ $S_{X}$, with $x^{\prime} \neq \pm x$. From Mankiewicz Theorem we know that any onto isometry $\tau: S_{X} \rightarrow S_{Y}$ is affine on $\left[x, x^{\prime}\right]$ and by Tingley's Theorem, $\left\{\tau(x), \tau\left(x^{\prime}\right)\right\}$ is a basis of $Y$. Taking coordinates with respect to these bases, we have $(\lambda, 1-\lambda) \in S_{X}$, also $(\lambda, 1-\lambda) \in S_{Y}$ and, moreover, $\tau(\lambda, 1-\lambda)=(\lambda, 1-\lambda)$ for every $\lambda \in[0,1]$. So, $\tau$ is linear on $\left[x, x^{\prime}\right]$ and now the result is clear from Theorem 3.7 .

Before we proceed with the proof of Theorem 3.11, we need this auxiliary result:

Proposition 3.9. Let $\left(X,\|\cdot\|_{X}\right)$ be a strictly convex normed space, $x \in X$ and $U \subset S_{X}$ a relative open subset. There exists $V \subset X$, an open neighbourhood of $x$, such that every point in $V$ is determined by its distances to the points in $U$, i.e., if $y, y^{\prime} \in V$ are such that $\|u-y\|_{X}=\left\|u-y^{\prime}\right\|_{X}$ for every $u \in U$, then $y=y^{\prime}$.

Proof. Suppose on the contrary that there are two sequences $\left(y_{n}\right),\left(y_{n}^{\prime}\right)$ that converge to $x$ and such that $U \subset \operatorname{Bis}\left(y_{n}, y_{n}^{\prime}\right)$ for every $n$.

We may suppose $x \notin \bar{U}$, the other case is obvious. Thus, the map

$$
u \in U \mapsto \sigma(u)=(u-x) /\|u-x\|_{X} \in S_{X}
$$

is well-defined and continuous - even Lipschitz, actually. As $X$ is strictly convex, no line has more than two points in common with $S_{X}$, so $\sigma$ is nearly an injective map. Namely, for each $y \in S_{X}$, there are at most two points 
whose image is $y$ and, moreover, if $\sigma(u)=\sigma\left(u^{\prime}\right)$, then $\sigma$ is injective in a relative neighbourhood of $u$.

So, $\sigma(U)$ contains a relative open subset of $S_{X}$, and this implies that $x$ is interior to the convex hull of $U \cup(2 x-U)$. This implies that also $y_{n}, y_{n}^{\prime}$ are interior to it for big $n$.

But every bisector is symmetric with respect to the middle point of the segment, i.e., $z \in \operatorname{Bis}\left(y_{n}, y_{n}^{\prime}\right)$ if and only if $y_{n}+y_{n}^{\prime}-z \in \operatorname{Bis}\left(y_{n}, y_{n}^{\prime}\right)$. Indeed, $\left\|z-y_{n}\right\|_{X}=\left\|z-y_{n}^{\prime}\right\|_{X}$ implies $\left\|y_{n}+y_{n}^{\prime}-z-y_{n}\right\|_{X}=\left\|y_{n}+y_{n}^{\prime}-z-y_{n}^{\prime}\right\|_{X}$, so the symmetry follows. Taking into account that $\left(y_{n}\right) \rightarrow x,\left(y_{n}^{\prime}\right) \rightarrow x$ and $\left(y_{n}+y_{n}^{\prime}\right) / 2 \rightarrow x$, it is clear that, for big $n$, we will have both $y_{n}$ and $y_{n}^{\prime}$ in the convex hull of

$$
\operatorname{Bis}\left(y_{n}, y_{n}^{\prime}\right) \cup\left(\left(y_{n}+y_{n}^{\prime}\right)-\operatorname{Bis}\left(y_{n}, y_{n}^{\prime}\right)\right)=\operatorname{Bis}\left(y_{n}, y_{n}^{\prime}\right)
$$

This means that there are $\lambda \in[0,1]$ and $u, u^{\prime} \in \operatorname{Bis}\left(y_{n}, y_{n}^{\prime}\right)$ such that $y_{n}=$ $\lambda u+(1-\lambda) u^{\prime}$. We may rewrite this as:

$$
\left\|y_{n}-u\right\|_{X}=\left\|y_{n}^{\prime}-u\right\|_{X},\left\|y_{n}-u^{\prime}\right\|_{X}=\left\|y_{n}^{\prime}-u^{\prime}\right\|_{X} \text { and } y_{n} \in\left[u, u^{\prime}\right] \text {. }
$$

As $\|\cdot\|_{X}$ is strictly convex, the points inside a segment are determined by its distances to the endpoints. Indeed, $\left\|y_{n}-u\right\|_{X}+\left\|y_{n}-u^{\prime}\right\|_{X}=\left\|u-u^{\prime}\right\|_{X}$ if and only if $y_{n} \in\left[u, u^{\prime}\right]$. So, we have $y_{n}^{\prime} \in\left[u, u^{\prime}\right]$, too. Moreover, $\lambda=$ $\left\|y_{n}-u\right\|_{X} /\left\|u-u^{\prime}\right\|_{X}$, so $y_{n}^{\prime}=y_{n}$ and we are done.

Remark 3.10. Our a priori impression was that there would be some result in the literature stating something like "The distances to a relative open subset of $S_{X}$ determine every point in $X$ whenever $\|\cdot\|_{X}$ is strictly convex". However, we have found nothing like this and, moreover, it seems much harder than expected to prove anything more general than Proposition 3.9. Actually, a result as the supposed-to-exist one would be enough for proving Conjecture 2.5.

Theorem 3.11. Let $\left(X,\|\cdot\|_{X}\right)$ be a strictly convex normed space, $\|\cdot\|_{X}^{\prime}$ an equivalent norm defined on $X$ and $\tau: S_{X} \rightarrow S_{X}^{\prime}$ an onto isometry. If the set of fixed points of $\tau$ has nonempty interior, then $\tau$ is the identity and $\|\cdot\|_{X}^{\prime}=\|\cdot\|_{X}$.

Proof. Let $F=\left\{x \in S_{X}: \tau(x)=x\right\}$ be the set of fixed points of $\tau$, we will denote its interior by $U$. As $\tau$ is continuous, $F$ is closed. We shall see that it is also relative open, so $F$ must be the whole sphere $S_{X}$. 
Suppose $e \in F$ and take $v \in U$. As $\|\cdot\|_{X}$ is strictly convex, the distances $\left\{\|e-u\|_{X}: u \in U\right\}$ determine $S_{X}$ in a neighbourhood of $(v-e) /\|v-e\|_{X}$, say $V$, so we have $\|w\|_{X}=\|w\|_{X}^{\prime}$ whenever $w /\|w\|_{X} \in V$. This means, obviously, that $V \subset S_{X}^{\prime}$, so we have $\|w-u\|_{X}^{\prime}=\|w-u\|_{X}$ for every $w$ and $u$ in (not necessarily relative) neighbourhoods of $e$ and $v$ respectively, i.e., $w \in e+\varepsilon B_{X}, u \in v+\varepsilon B_{X}$. As $\|\cdot\|_{X}$ and $\|\cdot\|_{X}^{\prime}$ are equivalent, the equality holds for every $w \in e+\delta B_{X}^{\prime}, u \in v+\delta B_{X}^{\prime}$ for some $\delta>0$. In particular, if $\|w\|_{X}=1$ and $w \in\left(e+\delta B_{X}^{\prime}\right) \cap\left(e+\varepsilon B_{X}\right)$, then $\|w-u\|_{X}^{\prime}=\|w-u\|_{X}=\|\tau(w)-u\|_{X}^{\prime}$ for every $u \in S_{X} \cap\left(v+\varepsilon B_{X}\right)=S_{X}^{\prime} \cap\left(v+\varepsilon B_{X}^{\prime}\right)$. By Proposition [3.9, we are done.

Theorem 3.12. Let $\left(X,\|\cdot\|_{X}\right)$ and $\left(Y,\|\cdot\|_{Y}\right)$ be finite dimensional strictly convex normed spaces and $\tau: S_{X} \rightarrow S_{Y}$ an onto isometry between their unit spheres. If there is a relative open $U \subset S_{X}$ where $\tau$ is linear, then $\tau$ is linear on $S_{X}$.

Proof. We just need to identify $\left(X,\|\cdot\|_{X}\right)$ and $\left(Y,\|\cdot\|_{Y}\right)$ with the corresponding $\mathbb{R}^{n}$ and apply Theorem 3.11

\section{Final examples and remarks}

This final section includes some less general results that, however, illustrate to which extent our approach can work. The end of the section includes a subsection where we introduce the notion of normed curvature.

\subsection{Absolute norms in the plane}

Throughout this subsection we will restrict ourselves to the case in which $\|\cdot\|_{X},\|\cdot\|_{Y}$ are absolute, normalized, norms on $\mathbb{R}^{2}$. Please observe that this implies that the symmetries with respect to the axes are linear isometries in both $\left(\mathbb{R}^{2},\|\cdot\|_{X}\right)$ and $\left(\mathbb{R}^{2},\|\cdot\|_{Y}\right)$.

Proposition 4.1. Let $\tau: S_{X} \rightarrow S_{Y}$ be an isometry and suppose that the only isometries of $S_{X}$ are $\pm \operatorname{Id}_{X}, \pm \phi$, where $\operatorname{Id}_{X}$ is the identity and $\phi$ is the symmetry with respect to the horizontal axis. Then $\tau$ is linear and, furthermore, if $\tau$ is not the identity, then it is one of the following maps:

- The rotation of angle $\pi / 2, \pi$ or $3 \pi / 2$ around the origin.

- The symmetry with respect to one of the following lines:

$$
\langle(1,0)\rangle,\langle(0,1)\rangle,\langle(1,1)\rangle,\langle(1,-1)\rangle \text {. }
$$


Proof. Let $\tau$ be such an isometry. The group of isometries of $S_{Y}$ is isomorphic to that of $S_{X}$, namely its isometries are $\pm \operatorname{Id}_{Y}$ and $\pm \psi$, where $\psi=\tau \circ \phi \circ \tau^{-1}$. As the symmetries with respect to the axes are also isometries of $Y, \psi$ must be one of these symmetries.

In particular, the fixed points of $\psi$ are $\pm(1,0)$ or $\pm(0,1)$ and the fixed points of $\phi$ are $\pm(1,0)$ and this means that $\tau(1,0)$ is either $(1,0),(0,1),(-1,0)$ or $(0,-1)$.

Now, we may suppose that $\left(X,\|\cdot\|_{X}\right)$ is strictly convex, the other case is an immediate consequence of Corollary [3.8. Suppose $\tau(1,0)=(1,0)$ and $\tau(0,1)=(0,1)$. If we show that $\tau=\mathrm{Id}$, then we are done because we can compose any of the other isometries with a linear isometry that makes the composition send $(1,0)$ to $(1,0)$ and $(0,1)$ to $(0,1)$.

Given any $(a, b) \in S_{X}$, with $a, b>0$, it is easy to determine the only $x \in S_{X}, x \neq(a, b)$ such that $\|(a, b)-(1,0)\|_{X}=\|x-(1,0)\|_{X}$. Namely, $x$ is $(a,-b)$, and the only $x^{\prime} \in S_{X}, x^{\prime} \neq(a, b)$ such that $\|(a, b)-(0,1)\|_{X}=$ $\left\|x^{\prime}-(0,1)\right\|_{X}$ is obviously $x^{\prime}=(-a, b)$. Please observe that these points are uniquely determined because of the strict convexity of $\left(X,\|\cdot\|_{X}\right)$ and that $\|(a, b)-x\|_{X}=2 b,\left\|(a, b)-x^{\prime}\right\|_{X}=2 a$. Now, if $\tau(a, b)=(c, d)$, then the Monotonicity Lemma implies that $c, d>0$ and the equality $\|(a, b)-$ $(1,0)\left\|_{X}=\right\| x-(1,0) \|_{X}$ implies $\|(c, d)-(1,0)\|_{Y}=\|\tau(x)-(1,0)\|_{Y}$. With the same argument than before, we obtain $\tau(x)=(c,-d)$, but $2 a=\|(a, b)-$ $x\left\|_{X}=\right\|(c, d)-\tau(x) \|_{Y}=2 c$, so $c=a$. By symmetry, we have also $d=b$, so $\tau=\mathrm{Id}$.

Remark 4.2. This statement could be seen as a cheat because it restricts the conclusion to norms for which we already know the group of isometries of its sphere. Of course, when we are trying to prove that the group of isometries of every sphere coincides with the group of linear isometries of the space, this may seem unfair.

However, it is easy to find some norms that fit in Proposition 4.1. Namely, if the points $\pm(1,0)$, or $\pm(0,1)$, are unique in $S_{X}$ in any intrinsic, metric, sense, then the sphere will have just the above referred isometries. Think, for example, in the norm whose unit sphere is a lens, see Figure 1. If we consider the point $(0,1)$, it is clear that it is unique in some sense, namely $(0,1)$ and $(0,-1)$ are the only points where $S_{X}$ is not a differentiable curve. Being a point of differentiability is not, to the best of our knowledge, something that can be said in terms of distances between points of $S_{X}$, but there is something similar than we can say. 

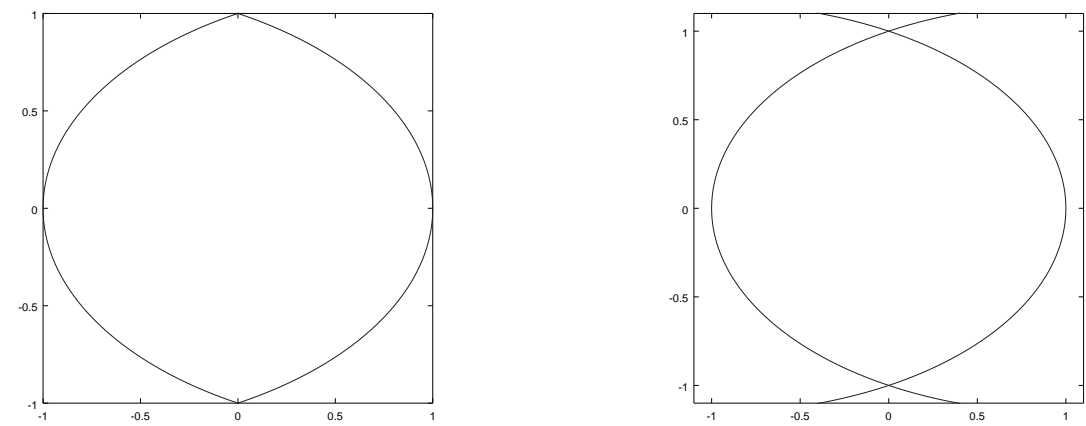

Figure 1: The unit sphere in Remark 4.2, whose associated ball is the intersection of two ellipses.

Namely, if we take for each point $x \in S_{X}$ and every $2>\delta>0$ the only points $a_{x}(\delta), a_{x}^{\prime}(\delta)$ such that

$$
\left\|x-a_{x}(\delta)\right\|_{X}=\left\|x-a_{x}^{\prime}(\delta)\right\|_{X}
$$

it is intuitively evident that the distances $\left\|a_{x}(\delta)-a_{x}^{\prime}(\delta)\right\|_{X}$ are smaller when $x=(0, \pm 1)$ than when $x$ is any other point in $S_{X}$. We shall not prove this, but the impression is that, when $\delta \rightarrow 0,\left\|a_{x}(\delta)-a_{x}^{\prime}(\delta)\right\|_{X} / \delta$ tends to 2 if $x \neq(0, \pm 1)$ and that this limit is smaller than 2 for $x=(0, \pm 1)$. And this is measured just by means of the distances between points in the sphere, this is closely related to Subsection 4.3.

Now, we deal with some quite more usual norms. Let us say, as in [29], that a norm $\|\cdot\|_{X}$ on $\mathbb{R}^{2}$ is symmetric when $\left\|\left(x_{1}, x_{2}\right)\right\|_{X}=\left\|\left(x_{2}, x_{1}\right)\right\|_{X}$ for every $x=\left(x_{1}, x_{2}\right) \in \mathbb{R}^{2}$. In the next result, we consider two norms $\|\cdot\|_{X},\|\cdot\|_{Y}$ in $\mathbb{R}^{2}$ with the following characteristics:

1. They are normalized.

2. They are absolute.

3. They are symmetric.

4. The only isometries $\phi: S_{X} \rightarrow S_{X}$ are the necessary for (2) and (3), i.e., the rotations of angle $0, \pi / 2, \pi$ and $3 \pi / 2$ and the symmetries with respect to the axis and the diagonals.

Corollary 4.3. With the above hypothesis, every isometry $\tau: S_{X} \rightarrow S_{Y}$ is linear and, moreover, it is either one of the isometries listed in (4) or one of them composed with the rotation of angle $\pi / 4$. 
Proof. This is very similar to Proposition 4.1.

Remark 4.4. This Corollary may seem superfluous, but it has some interesting consequences. Namely, this result applies for norms having $(1,0)$ and $(0,1)$ as interchangeable special points, like any $p$-norm in $\mathbb{R}^{2}$. If $p>2$ and $\|\cdot\|_{X}=\|\cdot\|_{p}$, then $(1,0)$ and $(0,1)$ are the isosceles orthogonal points in $S_{X}$ with least distance between them. So, every isometry $\phi: S_{X} \rightarrow S_{X}$ must send $(1,0)$ to $\pm(1,0)$ or $\pm(0,1)$. The opposite happens when $p<2$ : $(1,0)$ and $(0,1)$ have the greatest distance between isosceles orthogonal points in the sphere. This means that every $\|\cdot\|_{p}$ fulfils the hypotheses of the Corollary.

\subsection{A three-dimensional example}

Here we present an example of how our approach can be meaningful also in three-dimensional spaces.

Lemma 4.5. Let $\|\cdot\|_{\text {hex }}$ be the norm on $\mathbb{R}^{2}$ whose unit sphere is the hexagon with vertices $\pm(1,0),\left( \pm \frac{1}{2}, \pm 1\right)$, i.e., $\|(a, b)\|_{\text {hex }}=\max \{|b|,|a|+|b| / 2\}$. Then, $\left(\mathbb{R}^{2},\|\cdot\|_{\text {hex }}\right)$ has the Mazur-Ulam Property.

Proof. This norm has six length-1 segments in it sphere, so this lemma follows immediately from Proposition 3.4.

Let us recall the definition of modulus of convexity, see [31], p. 328:

Definition 4.6. A normed linear space $B$ is called uniformly convex if for each $\varepsilon, 0<\varepsilon \leq 2$, there is a $\delta(\varepsilon)>0$ such that $\left\|b_{1}+b_{2}\right\| \leq 2(1-\delta(\varepsilon))$ if $\left\|b_{1}-b_{2}\right\| \geq \varepsilon$ and $\left\|b_{1}\right\|=\left\|b_{2}\right\|=1$; the function $\delta$ is called the modulus of convexity of $B$.

and the following result, Theorem 4.1 in the same outstanding work, where $\delta_{2}$ is the modulus of convexity of the Euclidean space, please observe that $\delta_{2}$ does not depend on the dimension of the space:

Theorem 4.7 (Day, [31, Theorem 4.1]). B is uniformly convex with a modulus of convexity satisfying the inequality $\delta(\varepsilon) \geq \delta_{2}(\varepsilon)$ for $0<\varepsilon \leq 2$ if and only if $B$ is an inner-product space and $\delta$ is identically equal to $\delta_{2}$.

Since the modulus of convexity is defined just by means of some distances between points in the unit sphere, the characterisation of the Euclidean norm given by Day gives us: 
Corollary 4.8. Every $\mathbb{R}^{n}$ endowed with the Euclidean norm has the MazurUlam Property.

Corollary 4.8 is not new, it can be found both in [19] and [5].

This Lemma will come in handy for the proof of Example 4.10.

Lemma 4.9. Let $y_{1}, y_{2}, y_{3} \in S_{Y}$ be such that $y_{i}=\operatorname{Bis}\left(y_{j}\right) \cap \operatorname{Bis}\left(y_{k}\right)$ whenever $\{i, j, k\}=\{1,2,3\}$. Then, they are linearly independent.

Proof. Suppose they are linearly dependent and consider the plane $H=$ $\left\langle y_{1}, y_{2}, y_{3}\right\rangle$. The restriction of $\|\cdot\|_{Y}$ to $H$ is again a norm, so we have a twodimensional normed space with three mutually isosceles orthogonal points in its unit sphere. This cannot happen because of [32], Corollary 2.4, so we are done.

Example 4.10. Let $\|\cdot\|_{X}$ be the norm on $\mathbb{R}^{3}$ whose unit sphere is the revolution around the $x$-axis of the hexagon with vertices $\pm(1,0),\left( \pm \frac{1}{2}, \pm 1\right)$. Then, $\left(\mathbb{R}^{3},\|\cdot\|_{X}\right)$ has the Mazur-Ulam Property.

Proof of the Example. We will not use the explicit form of $\|\cdot\|_{X}$, but it is

$$
\|(a, b, c)\|_{X}=\max \left\{\|(b, c)\|_{2},|a|+\|(b, c)\|_{2} / 2\right\} .
$$

Suppose that there exist $\left(Y,\|\cdot\|_{Y}\right)$ and an onto isometry $\tau: S_{X} \rightarrow S_{Y}$. Consider the vectors in the usual basis of $\mathbb{R}^{3}, e_{1}, e_{2}, e_{3} \in S_{X}$ and their images $y_{1}=\tau\left(e_{1}\right), y_{2}=\tau\left(e_{2}\right), y_{3}=\tau\left(e_{3}\right)$.

It is clear that $e_{3} \in \operatorname{Bis}\left(e_{1}\right) \cap \operatorname{Bis}\left(e_{2}\right)$ and $e_{2} \in \operatorname{Bis}\left(e_{1}\right)$, so Lemma 4.9 implies that $y_{1}, y_{2}, y_{3}$ are linearly independent. We may consider $Y$ endowed with the basis $\mathcal{B}_{Y}=\left\{y_{1}, y_{2}, y_{3}\right\}$ and we have, in coordinates,

$$
\tau(1,0,0)=(1,0,0), \tau(0,1,0)=(0,1,0), \tau(0,0,1)=(0,0,1) .
$$

Consider the symmetric bisector $\operatorname{Bis}\left(e_{1}\right)=\left\{\left(a_{1}, a_{2}, a_{3}\right) \in S_{X}: a_{1}=0\right\}$, it is isometric to the Euclidean unit sphere of $\mathbb{R}^{2}$. This also happens with

$$
C_{X}=\left\{x \in S_{X}:\|x-(1,0,0)\|_{X}=1\right\}=S_{X} \cap\left((1,0,0)+S_{X}\right)
$$

and $C_{Y}=\tau\left(C_{X}\right)$, and the latest agrees with $S_{Y} \cap\left((1,0,0)+S_{Y}\right)$.

Take $x \in C_{X}$ and $\tau(x)=(a, b, c) \in C_{Y}$. As its distance to $(1,0,0)$ is 1 , we have $(a-1, b, c) \in S_{Y}$. We also have $\|(a-1, b, c)-(-1,0,0)\|_{Y}=1$, and this means that $(a-1, b, c) \in \tau\left(-C_{X}\right)=-C_{Y}$. Now, as the only point in $-C_{X}$ 
at distance 1 from $x$ is $x-(1,0,0)$, it must be $\tau(x-(1,0,0))=(a-1, b, c) \in$ $-C_{Y}$. On the one hand, this means that the sphere $S_{Y}$ includes the segment $[(a, b, c),(a-1, b, c)]$. On the other hand, $S_{Y}$ must include also every other segment of the (planar) hexagon with vertices

$\{(1,0,0),(a, b, c),(a-1, b, c),(-1,0,0),(-a,-b,-c),(1-a,-b,-c),(1,0,0)\}$

and this hexagon is the image of the hexagon whose vertices are

$$
\{(1,0,0), x, x-(1,0,0),(-1,0,0),-x,(1,0,0)-x,(1,0,0)\} .
$$

In particular this implies that the restriction of $\tau$ to this last hexagon is linear, so for this restriction to be the identity it just need to have some fixed point $x^{\prime} \neq( \pm 1,0,0)$. This happens with both $(0,1,0)$ and $(0,0,1)$, so every point in, say, the horizontal and vertical hexagons is fixed. This implies that $\|(a, b, 0)\|_{Y}=\|(a, b, 0)\|_{X}$ and $\|(a, 0, c)\|_{Y}=\|(a, 0, c)\|_{X}$ for $a, b, c \in \mathbb{R}$, and so

$$
\begin{gathered}
\left\|(a, b, c)-\left(a^{\prime}, b^{\prime}, c\right)\right\|_{Y}=\left\|(a, b, c)-\left(a^{\prime}, b^{\prime}, c\right)\right\|_{X} \text { and } \\
\left\|(a, b, c)-\left(a^{\prime}, b, c^{\prime}\right)\right\|_{Y}=\left\|(a, b, c)-\left(a^{\prime}, b, c^{\prime}\right)\right\|_{X}
\end{gathered}
$$

for every $a, b, c, a^{\prime}, b^{\prime}, c^{\prime} \in \mathbb{R}$. Actually, the restrictions of $\|\cdot\|_{X}$ and $\|\cdot\|_{Y}$ to the vertical and horizontal planes agree with $\|\cdot\|_{\text {hex }}$.

Claim 4.11. Let $z=\left(0, z_{2}, z_{3}\right), z^{\prime}=\left(0, z_{2}^{\prime}, z_{3}^{\prime}\right) \in \operatorname{Bis}((1,0,0)) \cap S_{Y}$. Then $\frac{z-z^{\prime}}{\left\|z-z^{\prime}\right\|_{Y}}$ also belongs to $\operatorname{Bis}((1,0,0)) \cap S_{Y}$.

Proof of the claim: Let $z^{\prime \prime}=\left(0, z_{2}^{\prime \prime}, z_{3}^{\prime \prime}\right)=\frac{z-z^{\prime}}{\left\|z-z^{\prime}\right\|_{Y}}$. It belongs to the bisector $\operatorname{Bis}((1,0,0))$ if and only if it does not belong to any segment that contains $\pm(1,0,0)$ and it is the midpoint of its segment, and this is what we will see.

Let $t, t^{\prime} \in[-1 / 2,1 / 2]$. We have

$$
\left\|\left(t, z_{2}, z_{3}\right)-\left(t^{\prime}, z_{2}^{\prime}, z_{3}^{\prime}\right)\right\|_{Y}=\left\|\left(t^{\prime}, z_{2}, z_{3}\right)-\left(t, z_{2}^{\prime}, z_{3}^{\prime}\right)\right\|_{Y}
$$

and this implies that $\|\cdot\|_{Y}$ is symmetric in the segment

$$
\begin{gathered}
{\left[\left(-1, z_{2}-z_{2}^{\prime}, z_{3}-z_{3}^{\prime}\right),\left(1, z_{2}-z_{2}^{\prime}, z_{3}-z_{3}^{\prime}\right)\right], \text { i.e. }} \\
\left\|\left(t, z_{2}-z_{2}^{\prime}, z_{3}-z_{3}^{\prime}\right)\right\|_{Y}=\left\|\left(-t, z_{2}-z_{2}^{\prime}, z_{3}-z_{3}^{\prime}\right)\right\|_{Y} \text { for } t \in[0,1] .
\end{gathered}
$$

In particular, as $\lambda=\left\|\left(0, z_{2}-z_{2}^{\prime}, z_{3}-z_{3}^{\prime}\right)\right\|_{Y} \leq 2$, we have

$$
\left\|\left(1 / 2,\left(z_{2}-z_{2}^{\prime}\right) / \lambda,\left(z_{3}-z_{3}^{\prime}\right) / \lambda\right)\right\|_{Y}=\left\|\left(-1 / 2,\left(z_{2}-z_{2}^{\prime}\right) / \lambda,\left(z_{3}-z_{3}^{\prime}\right) / \lambda\right)\right\|_{Y} .
$$


This readily implies that the claim holds.

Let $V$ be the set of points in $S_{X} \cap \operatorname{Bis}\left(e_{1}\right)$ whose images' first coordinates vanish. The Claim implies that for every couple of points $x_{1}, x_{2} \in V$ there exists another point between them that belongs to $V$ too-namely, as $V$ is symmetric, $-x_{2} \in V$ and the inverse image of $\left(\tau\left(x_{1}\right)-\tau\left(-x_{2}\right)\right) / \| \tau\left(x_{1}\right)+$ $\tau\left(x_{2}\right) \|_{Y}$ also belongs to $V$. As it is obvious that $V$ is closed, we have $V=$ $S_{X} \cap \operatorname{Bis}\left(e_{1}\right)$, and so $S_{Y} \cap \operatorname{Bis}\left(y_{1}\right)=S_{Y} \cap\{(0, b, c): b, c \in \mathbb{R}\}$. So, $S_{Y} \cap \operatorname{Bis}\left(y_{1}\right)$ is the intersection of a plane and the unit sphere and it is isometric to the two-dimensional Euclidean sphere. This, along with Corollary 4.8 implies that it is the two-dimensional Euclidean sphere. As $\tau(0,1,0)=(0,1,0)$ and $\tau(0,0,1)=(0,0,1)$, we conclude that $\tau$ is the identity on $\operatorname{Bis}\left(e_{1}\right)$ and this implies that it is the identity on $S_{X}$, so it is the restriction of a linear isometry.

Remark 4.12. Please observe that, whenever $\left(X,\|\cdot\|_{X}\right)$ is not strictly convex, there is some nonlinear isometric embedding $\mathbb{R} \rightarrow X$ (see [33]). If we still consider $\mathbb{R}^{3}$ endowed with the norm defined in Example 4.10, we have a nonlinear isometric embedding $i:\left(\mathbb{R}^{2},\|\cdot\|_{2}\right) \rightarrow\left(\mathbb{R}^{3},\|\cdot\|_{X}\right)$, where

$$
i\left(x_{1}, x_{2}\right)=\left(f\left(x_{1}, x_{2}\right), x_{1}, x_{2}\right) \text { and } f\left(x_{1}, x_{2}\right)=1 / 2\left\|\left(x_{1}, x_{2}\right)-\left(x_{1}^{\prime}, x_{2}^{\prime}\right)\right\|_{2} \text {. }
$$

Actually, any $f:\left(\mathbb{R}^{2},\|\cdot\|_{2}\right) \rightarrow \mathbb{R}$ such that $f(0,0)=0$ and

$$
f\left(x_{1}, x_{2}\right)-f\left(x_{1}^{\prime}, x_{2}^{\prime}\right) \leq 1 / 2\left\|\left(x_{1}, x_{2}\right)-\left(x_{1}^{\prime}, x_{2}^{\prime}\right)\right\|_{2}
$$

for every $\left(x_{1}, x_{2}\right),\left(x_{1}^{\prime}, x_{2}^{\prime}\right) \in \mathbb{R}^{2}$ would have done the trick, this example is just a slight modification of the last one in the same paper [33].

On the other hand, in the same work it is proved that every isometric embedding $\left(Y,\|\cdot\|_{Y}\right) \rightarrow\left(X,\|\cdot\|_{X}\right)$ is affine when $X$ is strictly convex. It seems that strict convexity can be key for the existence of nonlinear embeddings also in the spheres setting, so Baker's results led us to consider the following:

Conjecture 4.13. $X$ is strictly convex if and only if every isometric embedding $S_{Y} \rightarrow S_{X}$ extends linearly.

\subsection{A normed curvature}

Before we end this paper, we need to point out a minor result that may lead to some interesting questions. Throughout this work, we have tried to show that a very important thing to have in mind when dealing with Tingley's 
Problem is the concept of intrinsic metric property. We have been able to convert Day's Theorem 4.7 into a Mazur-Ulam Property statement just by taking into account that the Theorem was stated by means of distances between points in the unit sphere. Here we present a kind of generalisation of the curvature of a planar curve to curves in normed spaces, somehow in the spirit of Clarkson's modulus of Convexity:

Definition 4.14. Let $\left(X,\|\cdot\|_{X}\right)$ be a normed space, $\gamma:[0,1] \rightarrow X$ a curve and $x=\gamma(t) \in X$ for some $0<t<1$. Suppose that there is some $c>0$ such that $\gamma \cap\left(x+\delta S_{X}\right)$ contains exactly two points for every $0<\delta<c$. We define the curvature of $\gamma$ at $x$ measured with $\|\cdot\|_{X}$ as the following limit, whenever it exists:

$$
\mathcal{K}_{\|\cdot\|_{X}}^{\gamma}(x)=\sqrt{\lim _{\delta \rightarrow 0} \frac{\delta-\left\|a-a^{\prime}\right\|_{X} / 2}{(\delta / 2)^{3}}}=2 \sqrt{\lim _{a, a^{\prime} \rightarrow x} \frac{2\|x-a\|_{X}-\left\|a-a^{\prime}\right\|_{X}}{\left(\|x-a\|_{X}\right)^{3}}}
$$

where $a \neq a^{\prime}$ are the only points in $\gamma$ such that $\|x-a\|_{X}=\left\|x-a^{\prime}\right\|_{X}=\delta$.

Remark 4.15. If the space $X$ is two-dimensional and the curve is its unit sphere, then the $c$ in Definition 4.14 can always be chosen to be any $0<c<2$.

Besides, this curvature features some desirable characteristics:

- It is defined just by means of the norm and the curve, so it is generalizable to curves in arbitrary normed spaces.

- It is defined locally, or even infinitesimally.

- It is isometrically invariant.

- It is positively antihomogeneous with respect to the norm, i.e.,

$$
\mathcal{K}_{\lambda\|\cdot\|_{X}}^{\gamma}(x)=\frac{1}{\lambda} \mathcal{K}_{\|\cdot\|_{X}}^{\gamma}(x)
$$

for every $\lambda>0$.

$\mathcal{K}$ has also this important feature:

Theorem 4.16. This definition includes the notion of the curvature of a sphere in $\left(\mathbb{R}^{2},\|\cdot\|_{2}\right)$, i.e., $\mathcal{K}_{\|\cdot\|_{2}}^{x+\lambda S_{2}}\left(x^{\prime}\right)=1 / \lambda$ for every $x \in \mathbb{R}^{2}, \lambda>0$ and $x^{\prime} \in x+\lambda S_{2}$. 
Proof. The first we are going to show is that the curvature of the twodimensional Euclidean sphere at every point measured with the Euclidean norm is 1 . Let $x \in S_{2}$.

It is clear from (11) that our definition of curvature only depends on the distances, so every isometry must preserve the curvature. In particular, as $S_{2}$ is isometrically homogeneous, this means that its curvature is constant so we may suppose $x=(1,0)$.

The points near $(1,0)$ are $(\cos (t), \sin (t))$ for $t \in[-\varepsilon, \varepsilon]$, and the points $a, a^{\prime}$ in (11) are $a=(\cos (t), \sin (t)), a^{\prime}=(\cos (t),-\sin (t))$ for some positive $t$. We need to evaluate the right hand expression in (1). We have

$$
\begin{aligned}
& \mathcal{K}_{\|\cdot\|_{2}}^{S_{2}}((1,0))=2 \sqrt{\lim _{a, a^{\prime} \rightarrow x} \frac{2\|x-a\|_{X}-\left\|a-a^{\prime}\right\|_{X}}{\left(\|x-a\|_{X}\right)^{3}}}= \\
& =2 \sqrt{\lim _{t \rightarrow 0} \frac{2\|(1,0)-(\cos (t), \sin (t))\|_{2}-\|(\cos (t), \sin (t))-(\cos (t),-\sin (t))\|_{2}}{\left(\|(1,0)-(\cos (t), \sin (t))\|_{2}\right)^{3}}}= \\
& =2 \sqrt{\lim _{t \rightarrow 0} \frac{2 \sqrt{2-2 \cos (t)}-2 \sin (t)}{\sqrt{(2-2 \cos (t))^{3}}}}=\sqrt{8} \sqrt{\lim _{t \rightarrow 0} \frac{\sqrt{2-2 \cos (t)}-\sin (t)}{\sqrt{(2-2 \cos (t))^{3}}}} .
\end{aligned}
$$

For the sake of clarity, we are going to leave the limit as simple as we can. What we will actually compute is

$$
\lim _{t \rightarrow 0} \frac{\sqrt{2-2 \cos (t)}-\sin (t)}{\sqrt{(2-2 \cos (t))^{3}}}
$$

we need to show that it equals $1 / 8$. In the remaining of the proof we will avoid the $t \rightarrow 0$ and will not recall that $t>0$. As we will make heavy use of L'Hôpital's Rule, we will write as $\stackrel{*}{=}$ the equalities given by this Rule.

We will need this later:

$$
\lim _{t \rightarrow 0} \frac{\sin (t)}{\sqrt{2-2 \cos (t)}}=1 \text {. }
$$

This limit is 1 if and only if

$$
1=\lim \frac{\sin ^{2}(t)}{2-2 \cos (t)} \stackrel{*}{=} \lim \frac{2 \sin (t) \cos (t)}{2 \sin (t)}=\lim \cos (t),
$$


so (3) holds.

It is clear that in (2) both the numerator and the denominator tend to 0 . In the following there is one case where some explanation is needed, but we will leave the explanation for the end of the proof.

$$
\begin{aligned}
& \lim \frac{\sqrt{2-2 \cos (t)}-\sin (t)}{\sqrt{(2-2 \cos (t))^{3}}} \stackrel{*}{=} \lim \frac{\sin (t) / \sqrt{2-2 \cos (t)}-\cos (t)}{3 \sin (t) \sqrt{2-2 \cos (t)}}= \\
& =\lim \frac{\sin (t)-\cos (t) \sqrt{2-2 \cos (t)}}{3 \sin (t)(2-2 \cos (t))} \stackrel{*}{=} \\
& =\frac{1}{3} \lim \frac{\cos (t)-\sin (t) \cos (t) / \sqrt{2-2 \cos (t)}+\sin (t) \sqrt{2-2 \cos (t)}}{2 \sin ^{2}(t) \cos (t)(2-2 \cos (t))} * \\
& =\frac{1}{3} \lim \frac{-\sin (t)+\sqrt{2-2 \cos (t)} \cos (t)+\frac{2 \sin ^{2}(t)-\cos (t)}{\sqrt{2-2 \cos (t)}}+\frac{\cos (t) \sin ^{2}(t)}{\sqrt{2-2 \cos (t)}^{3}}}{2 \sin 2(t)(4 \cos (t)-1)} * \\
& =\frac{1}{3} \lim \frac{-\cos (t)+\frac{\sin (t) \cos (t)}{\sqrt{2-2 \cos (t)}}\left[2+\frac{\cos (t)}{2-2 \cos (t)}-\frac{3 \sin ^{2}(t)}{(2-2 \cos (t))^{2}}+1+\frac{2 \cos (t)}{2-2 \cos (t)}+4\right]}{-8 \sin ^{2}(t)+8 \cos ^{2}(t)-2 \cos (t)}+ \\
& +\frac{1}{3} \lim \frac{-3\left(\frac{\sin (t)}{\sqrt{2-2 \cos (t)}}\right)^{3}-\sin (t) \sqrt{2-2 \cos (t)}}{-8 \sin ^{2}(t)+8 \cos ^{2}(t)-2 \cos (t)}=
\end{aligned}
$$

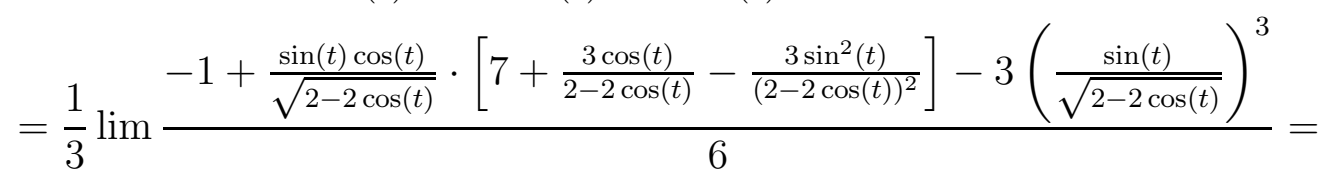

$$
\begin{aligned}
& =\lim \frac{\frac{\sin (t) \cos (t)}{\sqrt{2-2 \cos (t)}}\left(\frac{\cos (t)}{2-2 \cos (t)}-\frac{\sin ^{2}(t)}{(2-2 \cos (t))^{2}}\right)+1}{6} .
\end{aligned}
$$

So, what we need right now is to show that

$$
\begin{aligned}
-\frac{1}{4} & =\lim \frac{\sin (t) \cos (t)}{\sqrt{2-2 \cos (t)}}\left(\frac{\cos (t)}{2-2 \cos (t)}-\frac{\sin ^{2}(t)}{(2-2 \cos (t))^{2}}\right)= \\
& =\lim \frac{\cos (t)(2-2 \cos (t))-\sin ^{2}(t)}{(2-2 \cos (t))^{2}}=\lim \frac{2 \cos (t)-2 \cos ^{2}(t)-\sin ^{2}(t)}{4+4 \cos ^{2}(t)-8 \cos (t)}= \\
& =\lim \frac{2 \cos (t)-\cos ^{2}(t)-1}{4+4 \cos ^{2}(t)-8 \cos (t)}=\lim \frac{-\left(1-2 \cos (t)+\cos ^{2}(t)\right)}{4\left(1-2 \cos (t)+\cos ^{2}(t)\right)}
\end{aligned}
$$


so we have proved that (2) holds. The last application of L'Hôpital's Rule is the only tricky one, here we show that the numerator tends to 0 . The only problem could be here:

$$
\lim \left(\frac{2 \sin ^{2}(t)-\cos (t)}{\sqrt{2-2 \cos (t)}}+\frac{\cos (t) \sin ^{2}(t)}{\sqrt{2-2 \cos (t)}^{3}}\right)
$$

As we have (3), this reduces to

$$
\begin{aligned}
& \lim \left(\frac{-\cos (t)}{\sqrt{2-2 \cos (t)}}+\frac{\cos (t) \sin ^{2}(t)}{\sqrt{2-2 \cos (t)}}\right)=\lim \left(\cos (t) \frac{-(2-2 \cos (t))+\sin ^{2}(t)}{\sqrt{2-2 \cos (t)}}\right) \stackrel{*}{=} \\
& =\lim \frac{2 \sin (t) \cos (t)-2 \sin (t)}{3 \sin (t) \sqrt{2-2 \cos (t)}}=\lim \frac{2 \cos (t)-2}{3 \sqrt{2-2 \cos (t)}} \stackrel{*}{=} \lim \frac{-2 \sin (t)}{3 \sin (t) / \sqrt{2-2 \cos (t)}}
\end{aligned}
$$

and the last limit is just

$$
-\frac{2}{3} \sqrt{2-2 \cos (t)} \rightarrow 0
$$

so the first part of the proof is finished.

The usual curvature of $\lambda S_{2}$ is $1 / \lambda$ at every point for each $\lambda>0$ and, as the formula given for $\mathcal{K}_{\|\cdot\|_{X}}^{\gamma}$ is clearly positively antihomogeneous, it is clear that

$$
\mathcal{K}_{\|\cdot\|_{2}}^{\lambda S_{2}}(\lambda x)=\frac{1}{\lambda}
$$

for every $x \in S_{2}$, so both curvatures agree at every point of any centred sphere. Both are translation invariant, so they agree at every sphere and we have finished the proof.

\section{Aknowledgments}

Supported in part by DGICYT project MTM2016 $\cdot 76958 \cdot C 2 \cdot 1 \cdot P$ (Spain) and Junta de Extremadura programs GR·15152 and IB·16056. 


\section{References}

\section{References}

[1] D. Tingley, Isometries of the unit sphere, Geometriae Dedicata 22 (3) (1987) 371-378. doi:10.1007/BF00147942.

URL https://doi.org/10.1007/BF00147942

[2] S. Mazur, S. Ulam, Sur les transformations isométriques d'espaces vectoriels, normés, Comptes rendus hebdomadaires des séances de l'Académie des sciences 194 (1932) 946-948. URL https://gallica.bnf .fr/ark:/12148/bpt6k31473/f950.image. langFR

[3] L. Cheng, Y. Dong, On a generalized Mazur-Ulam question: Extension of isometries between Journal of Mathematical Analysis and Applications 377 (2) (2011) 464 - 470. doi:https://doi.org/10.1016/j.jmaa.2010.11.025. URL http://wwW.sciencedirect.com/science/article/pii/S0022247X10009601

[4] P. Mankiewicz, On extension of isometries in normed linear spaces, Bulletin de l'Académie Polonaise des Sciences, Série des Sciences Mathématiques, Astronomiques, et Physiques 20 (1972) 367 - 371.

[5] J. Becerra Guerrero, M. Cueto-Avellaneda, F. J. Fernández-Polo, A. Peralta, On the extension of isometries between the unit spheres of a JB*-triple and a Ban URL https://arxiv.org/abs/1808.01460v2

[6] M. Cueto-Avellaneda, A. M. Peralta, The Mazur-Ulam property for commutative von Neumann algebras, Linear and Multilinear Algebra (to appear)doi:10.1080/03081087.2018.1505823.

[7] G. G. Ding, The 1-Lipschitz mapping between the unit spheres of two Hilbert spaces can be extended to a real linear isometry of the whole space, Science in China Series A: Mathematics 45 (4) (2002) 479-483.

[8] G.-G. Ding, J.-Z. Li, Sharp corner points and isometric extension problem in Banach spaces, Journal of Mathematical Analysis and Applications 405 (1) (2013) 297

- 309. doi:https://doi.org/10.1016/j.jmaa.2013.04.002.

URL http://www.sciencedirect.com/science/article/pii/S0022247X13003119

[9] X. N. Fang, J. H. Wang, Extension of isometries between the unit spheres of normed space $E$ Acta Mathematica Sinica $22 \quad$ (6) (2006) 1819-1824. 
doi:10.1007/s10114-005-0725-z.

URL https://doi.org/10.1007/s10114-005-0725-z

[10] F. J. Fernández-Polo, J. J. Garcés, A. M. Peralta, I. Villanueva, Tingley's problem for spaces of trace class operators, Linear Algebra and its Applications 529 (2017) 294 - 323. doi:https://doi.org/10.1016/j.laa.2017.04.024. URL http://www.sciencedirect.com/science/article/pii/S0024379517302616

[11] F. J. Fernández-Polo, $\quad$ A. M. Peralta, Tingley's problem through the facial structure of an atomic JBW*-triple, Journal of Mathematical Analysis and Applications 455 (1) (2017) 750 - 760. doi:https://doi.org/10.1016/j.jmaa.2017.06.002. URL http://www.sciencedirect.com/science/article/pii/S0022247X17305528

[12] F. J. Fernández-Polo, A. Peralta, On the extension of isometries between the unit spheres of a $\mathrm{C}^{*}$-algebra and $B(H)$, Trans. Amer. Math. Soc. Ser. B 5 (3) (2018) 63-80. doi:10.1090/btran/21.

[13] F. J. Fernández-Polo, A. M. Peralta, Low rank compact operators and Tingley's problem, Advances in Mathematics $338 \quad(2018) \quad 1 \quad-40$. doi:https://doi.org/10.1016/j.aim.2018.08.018. URL http://www.sciencedirect.com/science/article/pii/S0001870818303311

[14] F. J. Fernández-Polo, A. Peralta, On the extension of isometries between the unit spheres of von Neumann algebras, Journal of Mathematical Analysis and Applications 466 (1) (2018) 127-143.

[15] A. Jiménez-Vargas, A. Morales-Campoy, A. M. Peralta, M. I. Ramírez, The Mazur-Ulam property for the space of complex null sequences, Linear and Multilinear Algebra 67 (4) (2019) 799-816. doi:10.1080/03081087.2018.1433625.

[16] V. Kadets, M. Martín, Extension of isometries between unit spheres of finite-dimensional poly Journal of Mathematical Analysis and Applications 396 (2) (2012) 441

- 447. doi:https://doi.org/10.1016/j.jmaa.2012.06.031. URL http://www.sciencedirect.com/science/article/pii/S0022247X12005203 
[17] J.-Z. Li, Mazur-Ulam property of the sum of two strictly convex Banach spaces, Bulletin of the Australian Mathematical Society 93 (3) (2016) 473-485. doi:10.1017/S0004972715001215.

[18] M. Mori, Tingley's problem through the facial structure of operator algebras, Journal of Mathematical Analysis and Applications 466 (2) (2018) 1281-1298. doi:https://doi.org/10.1016/j.jmaa.2018.06.050

[19] M. Mori, N. Ozawa, Mankiewicz's theorem and the mazur-ulam property for $\mathrm{c}^{*}$-algebras, Studia Mathematica to appear.

[20] A. M. Peralta, A survey on tingley's problem for operator algebras, Acta Sci. Math. (Szeged) 84 (1-2) (2018) 81-123.

[21] A. M. Peralta, Extending surjective isometries defined on the unit sphere of $\ell_{\infty}(\Gamma)$, Rev. Mat. Complut. 32 (1) (2019) 99-114.

[22] A. M. Peralta, R. Tanaka, A solution to Tingley's problem for isometries between the unit sph Science China Mathematics 62 (3) (2019) 553-568. doi:10.1007/s11425-017-9188-6. URL https://doi .org/10.1007/s11425-017-9188-6

[23] D. Tan, R. Liu, A note on the Mazur-Ulam property of almost-CL-spaces, Journal of Mathematical Analysis and Applications 405 (1) (2013) 336 - 341. doi:https://doi.org/10.1016/j.jmaa.2013.03.024. URL http://www.sciencedirect.com/science/article/pii/S0022247X13002175

[24] R. Tanaka, A further property of spherical isometries, Bulletin of the Australian Mathematical Society 90 (2) (2014) 304-310. doi:10.1017/S0004972714000185.

[25] R. Tanaka, The solution of Tingley's problem for the operator norm unit sphere of complex $n$ Linear Algebra and its Applications 494 (2016) 274 - 285. doi:https://doi.org/10.1016/j.laa.2016.01.020. URL http://www.sciencedirect.com/science/article/pii/S0024379516000239

[26] R. Tanaka, Tingley's problem on finite von Neumann algebras, Journal of Mathematical Analysis and Applications 451 (1) (2017) 319 - 326. doi:https://doi.org/10.1016/j.jmaa.2017.02.013. URL http://www.sciencedirect.com/science/article/pii/S0022247X17301634 
[27] R. Wang, X. Huang, The Mazur-Ulam property for two-dimensional somewhere-flat spaces, Linear Algebra and its Applications 562 (2019) 55 - 62. doi:https://doi.org/10.1016/j.laa.2018.09.024. URL http://www.sciencedirect.com/science/article/pii/S0024379518304658

[28] H. Martini, K. J. Swanepoel, G. Weiss, The geometry of Minkowski spaces - A survey. Part I, Expositiones Mathematicae $19 \quad(2) \quad(2001) \quad 97 \quad-\quad 142$. doi:https://doi.org/10.1016/S0723-0869(01)80025-6. URL http://www.sciencedirect.com/science/article/pii/S0723086901800256

[29] R. Tanaka, Tingley's problem on symmetric absolute normalized norms on $\mathrm{R}^{2}$, Acta Mathematica Sinica, English Series 30 (8) (2014) 1324-1340. doi:10.1007/s10114-014-3491-y. URL https://doi.org/10.1007/s10114-014-3491-y

[30] J. Cabello Sánchez, A. Gordillo-Merino, A characterisation of Euclidean normed planes via bisectors, Bulletin of the Australian Mathematical Society 99 (1) (2019) 121-129. doi:10.1017/S0004972715001215.

[31] M. M. Day, Some characterizations of inner-product spaces, Transactions of the American Mathematical Society 62 (2) (1947) 320-337. URL http://www.jstor.org/stable/1990458

[32] D. Ji, J. Li, S. Wu, On the uniqueness of isosceles orthogonality in normed linear spaces, Results in Mathematics $59 \quad$ (1) (2011) 157-162. doi:10.1007/s00025-010-0069-6. URL https://doi.org/10.1007/s00025-010-0069-6

[33] J. A. Baker, Isometries in normed spaces, The American Mathematical Monthly 78 (6) (1971) 655-658. URL http://www.jstor.org/stable/2316577 